\title{
Application of artificial neural networks in the process of drying CPC
}

\author{
Yunusov Bakhtiar ${ }^{1}$ \\ ${ }^{1}$ The Tashkent state technical university, after I. Karimov, Tashkent city, Uzbekistan

\begin{abstract}
Annotation Training of artificial neural networks using the experimental data obtained in the process of drying of cotton powdered cellulose (CPC), have shown that, experiments can be conducted on the computers, which solves many of the practical and ethical issues. Therefore, arose and remain at the present time, the objective of neural simulation of processes and create a computing system (artificial neural network)
\end{abstract}

\section{Introduction}

Neural networks (NN) are computational structures that model simple biological processes usually associated with the processes of the human brain. Adaptable and trainable, they are parallel systems capable of learning by analyzing positive and negative impacts. An elementary transformer in these networks is an artificial neuron or simply a neuron, so named by analogy with a biological prototype.

\section{Application of "Artificial Intelligence" in the field of drying technology}

In the modern world, human progress, development and productivity are practically achieved only when computers take on part of the intellectual load. One of the ways to achieve maximum progress in this area is "Artificial Intelligence", when the computer takes over not only the same type, repetitive operations, but also can learn itself. In addition, the creation of a full-fledged "Artificial Intelligence" opens up new horizons for human development. In this regard, the relevance of this work consists in the application of the intelligent program "Neural networks" in the field of heat power engineering. Specialists from fields like engineering design, power engineering, processions and apparatus, physiology and psychology, etc. intrigued by the possibilities offered by this technology and are looking for ways to apply them in their own directions.

The purpose of the study of artificial neural networks is the application of "Artificial Intelligence" in the field of drying technology. Teaching the program, automating experiments and solving complex problems. For example, they learn from experience or experimental data, generalize previous use cases to new cases, and extract significant properties from incoming information containing redundant data.
Artificial neural networks can change their behavior depending on the external environment. This factor, more than any other, is responsible for the interest they generate. Upon presentation of the input signals (possibly along with the required outputs), they self-adjust to provide the required response. Analysis of the literature has shown that many training algorithms have been developed, each with its own strengths and weaknesses. But experiments have shown that there are still problems as to what the network can learn and how the learning should be done.

Artificial neural networks have been proposed for tasks ranging from experiment control to data optimization. Potential applications are those where human intelligence is ineffective and conventional computation is cumbersome or inadequate. This class of applications is, at any rate, no less than the class served by conventional computing, and artificial neural networks can be expected to take their place alongside conventional computing as a complement of the same scope and importance. [1, 2, 3].

For drying dispersed materials in a number of industries, mechanized and low-performance drying machines are widely used devices. Their serious disadvantages are due to the discrepancy between the regime and design characteristics of the apparatus for drying a specific material [4].

More and more application in recent years is finding a progressive drying method in air - fountain dryers, which are free from most of the disadvantages inherent in fluidized bed dryers. The widespread introduction of these dryers was hampered by the lack of correct calculation methods and rational installation schemes that allow for effective drying of bulk materials. In this regard, the proposed calculation methods gave satisfactory results only in some cases.

The analysis of the obtained experiments on the study of the hydrodynamics of the layer in apparatuses for obtaining the regularities of processes depending on the design parameters, the method of feeding the liquefying agent, the properties and characteristic dimensions of the 
initial materials, etc. $\neg$ There is a method of supplying the coolant. The defining size for processing the research results is the equivalent diameter of the particles of the solid phase, and the defining flow rate is the speed of the heat carrier in a narrow section of the apparatus.

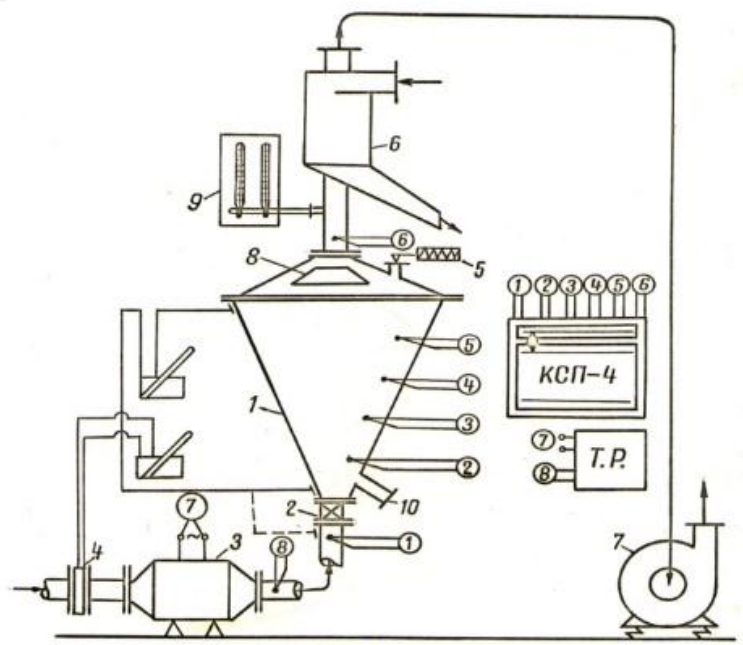

Figure I. Aerial drying apparatus for experiments.

1 - Camera; 2 - Swirler; 3 - Colorifier; 4 - diaphragm; 5 - Feeder; 6 - CDW; 7 - Fan; 8 - Window; 9 Psychrometer

The working process of many dryers is largely due to hydrodynamics, heat and mass transfer between interacting phases. The task of increasing the intensity of processes arose in connection with modern trends in the development of thermal power plants. Studies of methods of organizing the drying process in various devices have shown that twisting the flow is an extremely active means of intensifying the process of heat and mass transfer in general [5].

o determine the optimal modes of the drying process in the aerial drier (Fig. I.), hydrodynamic studies were carried out, the main purpose of which was to identify the effect of the height of the material layer, as well as the swirl angle of the gas flow on the velocity of the beginning of fluidization.

Therefore, a systematic study of the nature and boundaries of stable modes in this apparatus was carried out. Experiments carried out in a conical apparatus with transparent walls have shown that good mixing and grinding occur in the peripheral zone of the gushing layer.

Experiments to determine the pressure drop in the layer depending on its height were carried out on an experimental setup without swirling the flow. The layer resistance was determined from the pressure drop in the layer depending on the velocity of the fluidizing agent and was measured using impulse tubes connected to a micromanometer, each time changing the layer height by $0.05 \mathrm{~m}$. Experimentally obtained dependence of the layer hydraulic resistance on the velocity air is shown in Fig. 2.

The critical flow rate, or loss of bed stability, depends on the bed height, physical characteristics of the material and gas, as well as on the geometric parameters of the installation. The height of the layer was changed from $0.25 \mathrm{~m}$ to $0.55 \mathrm{~m}$. Analysis of experimental data shows that the most rational layer height is $0.35+0.40 \mathrm{~m}$. But at the initial moisture content of the material, the layer forms channels and closed zones, since the material is high-moisture and aggregation is peculiar to it. As free moisture is removed, the layer begins to move into a fluidized bed.

In order to destroy channels and stagnant zones in the chamber, we used a vane swirler for swirling the gas flow in the design of the aerial drier.

Experiments to determine the pressure drop in the layer depending on the swirl angle of the gas flow were carried out on an experimental setup (Fig. I.) using swirlers at different angles of inclination of the blades relative to the apparatus axis $\left(15^{\circ}, 25^{\circ}, 35^{\circ}\right)$ and at different layer heights. The resistance of the material layer was determined by the pressure drop in the layer depending on the flow rate of the coolant and measured with a micromanometer. Therefore, one of the impulse tubes was placed above the swirler, and the other above the free surface of the layer. In this case, the value of $\Delta P$ was measured on the manometer scale. The beginning of fluidization was assessed visually, the values of the dependence $\Delta \mathrm{P}=\mathrm{f}(\mathrm{v})$ were taken as the average of three measurements, differing from each other by no more than $5 \%$. The experimental results are presented in the graph in Fig. 3.

The use of a swirler in the design of an aerofoil apparatus for the destruction of the formed channels and stagnant zones in the chamber led to an increase in the resistance of the apparatus. The value of this resistance, depending on the angles of inclination of the swirler blades, and the resistance of the layer are of great importance for the choice of auxiliary equipment: fan, air heater, etc.

For teaching NN we have chosen the LevenbergMarquardt backpropagation method.

Distinguish between teaching neural networks with a teacher and without a teacher. In our case, we chose to teach NS with a teacher. This method gives, for each input vector, a target vector representing the desired output. Together they are called a learning pair. Usually the network is trained on a certain number of such training pairs. The vectors of the training set are presented sequentially, the errors are calculated, and the weights are adjusted for each

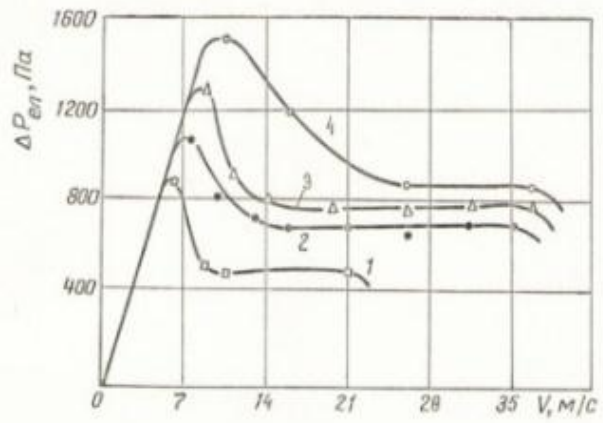

Fig. 2. Layer resistance without flow swirling at: 1 $\mathrm{H}=300 \mathrm{~mm}, 2$ - $350 \mathrm{~mm}, 3-400 \mathrm{~mm}, 4-450 \mathrm{~mm}$. 


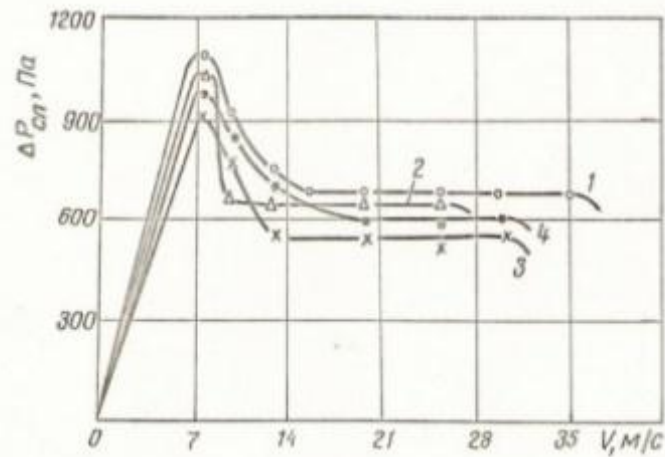

Fig. 3. Layer resistance at different swirlers: 1without swirling; $2-15^{\circ} ; 3-25^{\circ} ; 4-35^{\circ}$.

vector until the error throughout the training array reaches an acceptably low level.

After successfully teaching the neural network, we conducted computer experiments and obtained the following graphs (Fig. 4 - Fig. 5).

Algorithm for the functioning and learning of the neural network, the whole process can be written and then programmed in terms and using the operations of matrix algebra.

Apparently, such an approach will provide a faster and more compact implementation of the neural network than its implementation based on the concepts of objectoriented (OO) programming. Recently, however, it is the OO approach that has dominated, and often special OO languages are developed for programming $\mathrm{NN}$, although universal $\mathrm{OO}$ languages, for example, $\mathrm{C}++$ and Pascal, were created just to eliminate the need to develop any other $\mathrm{OO}$ languages, in whatever area they are going to apply.

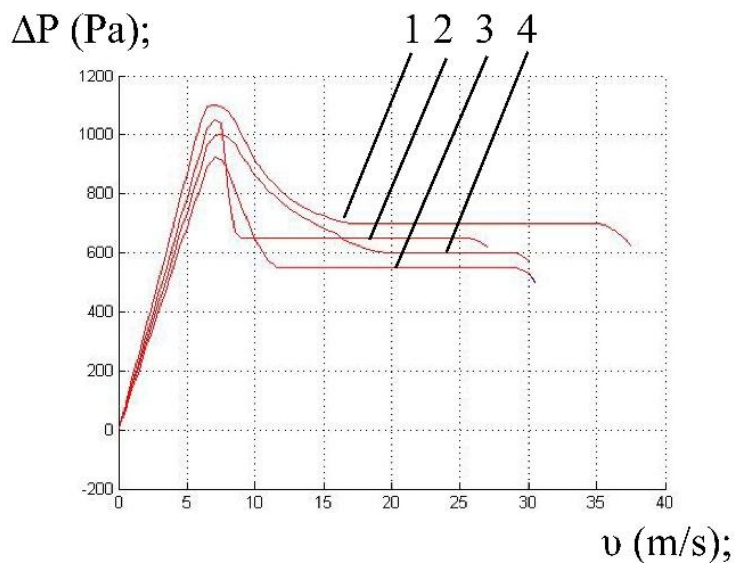

Fig. 4. Results of a computer experiment. $(\mathrm{H}=$ const, coolant swirling coal

$1-\alpha=\mathrm{O}{ }^{\circ} \mathrm{C} ; 2-15{ }^{\circ} \mathrm{C} ; 3-25^{\circ} \mathrm{C} ; 4-35^{\circ} \mathrm{C}$ from the axis of the apparatus).

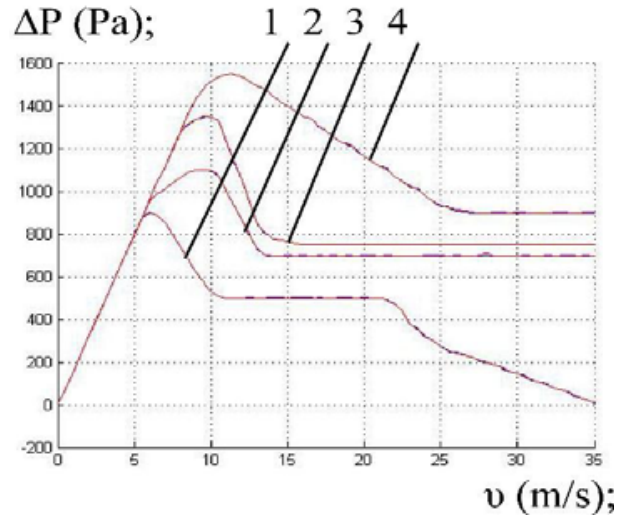

Fig. 5 Results of a computer experiment. $(\alpha=\mathrm{O} \circ \mathrm{C}=$ const, material layer $1-\mathrm{H}=300 \mathrm{~mm} ; 2$ $350 \mathrm{~mm} ; 3$ - $400 \mathrm{~mm} ; 4$ - $450 \mathrm{~mm}$ )

And yet, programming neural networks using the $\mathrm{OO}$ approach has its advantages:

First, it allows you to create a flexible, easily reconstructed hierarchy of neural network models.

Secondly, such an implementation is the most transparent for the programmer, and allows even nonprogrammers to design neural networks.

Third, the level of programming abstractness inherent in OO languages.

Analysis of the experimental results allows us to draw the following conclusions:

- Using the analysis of literary sources, an algorithm and methodology for training artificial intelligence (AI), a neural network (NS), "MatLab" was selected to study the hydrodynamics of an aerial type dryer.

- Experimental data on the study of the hydrodynamics of the apparatus and the layer in the drying chamber for teaching NS, "MatLab".

- Thus, after successful training of the neural network, we carried out computer experiments and obtained the following graphs (Fig. 4. - Fig. 5.) with an error of $0.0001 \%$ and below.

- the dependence of the pressure drop on the layer height shows that with an increase in the layer height, the critical flow rate increases and, accordingly, the range of operating speeds decreases;

- the dependence of the pressure drop of the layer on the swirl angle of the flow has a specific character. So, at a gas flow swirl angle of $25^{\circ}$, the minimum value of the pressure drop is observed, which is explained by the best mixing of the material layer.

\section{Literature}

1. V. V. Kruglov, V. V. Borisov. Artificial neural networks. Theory and practice. 2nd edition. Moscow. Hot line - Telecom. 2002 year

2. F. Wasserman. Neurocomputer technology. - M.: Mir, 1992 .-- $240 \mathrm{p}$.

3. V. V. Kruglov, V. V. Borisov, E. V. Khoritonov. Neural networks: Configurations, training, applications. - Smolensk: Publishing house of Moscow. energ. in-ta, fil-1 in Smolensk, 1998. 
4. L.G. Golubev, B.S.Sazhin, E.R. Valashek. Drying in the chemical and pharmaceutical industry. - M. : Medicine, 1978, p. 271

5. D. N. Mukhiddinov, B. P. Lukochevsky, B. Kh. Yunusov, A. I. Yufa, S. I. Yakubov. Simulation of the hydrodynamics of a gushing type dryer with grinding. - Izv. Academy of Sciences of UzSSR, series of technical. nauk, 1984, no. 4, p. 17-22.

6. Artikov A.A. Computer methods of analysis and synthesis of technological systems. Toshkent. 2012. from $204-208$.

7. Aliev R.A., Aliev R.R. The theory of intelligent systems. Baku. 2001.719 s. 